\title{
Co-culture of JEG-3, BeWo and syncBeWo cell lines with adrenal H295R cell line: an alternative model for examining endocrine and metabolic properties of the fetoplacental unit
}

\author{
Eliza Drwal · Agnieszka Rak • Ewa Gregoraszczuk
}

Received: 2 March 2017/ Accepted: 7 September 2017/Published online: 30 September 2017

(C) The Author(s) 2017. This article is an open access publication

\begin{abstract}
Monocultures of different placental cells are used for many physiological and toxicological studies; however, they are not a true reflection of the interaction between placenta and fetus. To develop the most appropriate model to study endocrine and metabolic properties of fetoplacental unit we used three co-culture models of placental cells nonfusogenic JEG-3, unsyncytialised BeWo (BeWo) and syncytialised BeWo (syncBeWo) cultured with adrenal (H295R) cells. As an end point of endocrine properties we investigated steroids receptors expression and steroid secretion, while as metabolic properties AhR, CYP1A1and COMT expression. Progesterone (P4), estradiol (E2) and human chorionic gonadotropin (hCG) secretion (ELISA) and 3ßHSD, CYP19, estrogen $(E R \alpha / \beta)$, progesterone (PR) and aryl hydrocarbon (AhR) receptors, CYP1A1 and COMT protein expression (Western blot) were evaluated. Comparing three co-culture models we observed: (1) there were no differences between JEG-3 and BeWo in the PR expression, however it was higher in BeWo
\end{abstract}

Electronic supplementary material The online version of this article (doi:10.1007/s10616-017-0142-z) contains supplementary material, which is available to authorized users.

E. Drwal · A. Rak · E. Gregoraszczuk $(\square)$

Department of Physiology and Toxicology of

Reproduction, Institute of Zoology and Biomedical

Research, Jagiellonian University in Krakow,

Gronostajowa 9, 30-387 Kraków, Poland

e-mail: ewa.gregoraszczuk@uj.edu.pl compared to syncBeWo; (2) there were no differences in $\mathrm{ER} \alpha$ protein expression in all models, while profile of ER $\beta$ expression was the highest in syncBeWo; (3) high $\mathrm{P} 4$ secretion in JEG-3 and BeWo while low in syncBeWo; (4) high E2 levels in JEG-3 and syncBeWo, while low E2 secretion in BeWo; (5) the highest hCG secretion in the JEG-3 and syncBeWo than in BeWo (6) the highest AhR, CYP1A1 and COMT expression in syncBeWo. Based on the results showing higher hCG secretion in the JEG-3 than in BeWo, representing villous and extravillous phenotype we suggest that JEG-3 model could be used to study fetoplacental steroidogenesis at the 1 st, while BeWo model at the 3rd. Results showing comparable profiles of AhR, CYP1A1 and COMT expression in JEG-3 and BeWo models and the significantly higher expression in synBeWo points to synBeWo as a good model for study the metabolic properties.

Keywords Placenta cell lines · H295R cells · Fetoplacental steroidogenesis · Fetoplacental metabolism $\cdot$ Receptors expression

\section{Introduction}

Cell-lines derived from human placenta and chorion, such as human choriocarcinoma cells BeWo or JEG-3 are an alternative for study the placenta function (Sullivan 2002). There are two main differentiation 
pathways in human placentas, villous (VCT) and extravillous (EVT) cytotrophoblast (Lee et al. 2016). BeWo represent placenta villous (Orendi et al. 2010), while JEG-3 extravillous cells (Lee et al. 2016). Microarray analysis has indicated that approximately 2700 genes are differentially expressed between BeWo and JEG-3 cells, suggesting that they are suited only for specific experimental paradigms. These differences in gene expression patterns suggest that JEG3 and BeWo cell lines will vary in their capacity to respond to an identical experimental treatment (Burleigh et al. 2007). JEG-3 lines are widely used to study the molecular mechanisms underlying the proliferation and invasive potential of cytotrophoblast, while BeWo cells are commonly used to study syncytialisation, adhesion and endocrine function (Hannan et al. 2010). Importantly, BeWo cell lines have fusogenic properties and, in culture with forskolin (a cyclic AMP inducer), are able to form syncytiotrophoblasts (Wice et al. 1990), thus making them a good third trimester placental model (Zachariades et al. 2011). Moreover, few papers indicate that significant differences in gene expression promotes fusion such as: $\beta$ subunit of human chorionic gonadotropin, placental alkaline phosphatase, LGALS13, syncytin-1 or syncytin- 2 in BeWo cells treatment with forskolin compared to non-treated BeWo cells (Orendi et al. 2010; Frendo et al. 2003).

In the complex scenario of the feto-maternal interface, endocrine, paracrine, and autocrine factors must be taken into consideration. Hormones, such as $\mathrm{E} 2, \mathrm{P} 4$ and $\mathrm{hCG}$ are the major players of the endocrine regulation that take place at the feto-maternal interface. hCG, which is the hormone produced by the trophoblast in the very early stages of pregnancy, plays a key role in making sure that the endometrium is ready to receive the embryo implantation (Tsampalas et al. 2010). The placenta has poor capacity of producing de novo estrogen (Escobar et al. 2011), therefore uses androgens such as dehydroepiandrosterone (DHEA), derived from the fetal and maternal adrenal glands to produce estrogen (Gude et al. 2004). Experimental models using independent cell lines alone do not reflect the interaction between placenta and fetus and provide only partial information concerning hormone secretion; moreover, when cultured alone, cell lines require supplementation with precursors for steroid secretion. The H295R human adrenocortical carcinoma cell line possesses all the enzymatic capacities of the undifferentiated fetal adrenal gland, and produces androgens, therefore providing fetal precursors for estrogen (Thibeault et al. 2014). The expression of PR in BeWo, syncBeWo and JEG-3 cell lines was described by Zachariades et al. (2011). Furthermore, two isoforms of ERs in BeWo cells (Jiang et al. 1997) and JEG-3 cells have also been demonstrated (Mehta et al. 2002).

Additionally, the placenta-expressed enzymes of phase I (i.e. CYP1A1) and phase II (i.e. catechol-Omethyl transferase, COMT) of metabolism are responsible for the detoxification of endogenous and exogenous hormones (Isoherranen and Thummel 2013; Hakkola et al. 1996a). CYP1A1 expression and activity have been detected in the first and third trimesters at both mRNA and protein levels (Hakkola et al. 1996b; Stejskalova and Pavek 2011; Czekaj et al. 2005). There are data showing that both placental (Hakkola et al. 1996b; Stejskalova et al. 2013) and adrenal (Bláha et al. 2006) CYP1A1 are regulated by AhR. COMT activity in the full-term placenta has been described by Barnea et al. (1988). To our knowledge there are no data showing COMT protein expression in placenta cell lines.

Coculture models including cells and/or tissues representative of both the fetus and the maternal counterpart have been developed to study a wider spectrum of interactions at the feto-maternal interface (Wang et al. 2012; Dunk et al. 2003; Helige et al. 2008; Moser et al. 2010). Such approaches mainly focus on the invasion of human placenta inside the maternal decidua. These coculture models are a powerful tool as they include all the main cell types involved at the feto-maternal interface. They nevertheless require synchronization of primary cultures from tissues which are sometimes difficult to obtain. In order to overcome these limitations, Mannelli et al. (2015) recently applied an in vitro system that could be helpful to study the molecular interactions at the fetomaternal interface even in laboratories that do not have availability of fresh tissues. The co-culture of $\mathrm{H} 295 \mathrm{R}$ and BeWo cells as a unique in vitro model to reproduce the steroidogenic cooperation between fetus and placenta during pregnancy have been characterized and proposed for use to study the endocrinedisrupting effects of environmental chemicals by Thibeault et al. (2014). As an end point authors analyzed progesterone, DHEA, androstenedione, estradiol, estriol, and estrone, CYP 19 activity and 
hCG production by $\mathrm{H} 295 \mathrm{R}$ and BeWo cells in monoculture or in co-culture over a $24 \mathrm{~h}$ period.

In the presented data we decided despite of BeWo cell line, to use JEG-3 cells with non-fusogenic properties and syncBeWo representing syncytiotrophoblast and additionally as an end point CYP19 and $3 \beta$-HSD protein expression, steroid receptors $(E R \alpha / \beta$, $\mathrm{PR}$ ) protein expression, while for metabolic properties we selected AhR, CYP1A1 and COMT protein expression.

\section{Materials and methods}

\section{Reagents}

DMEM/F12 medium, phosphate buffered saline (PBS), trypsin were purchased from Gibco Life Technologies (Paisley, United Kingdom). Fetal bovine serum (FBS, heat inactivated and stripped) was purchased from Biowest (Nuaillé, France). NuSerum and ITS + Premix were obtained from BD Biosciences (Mississauga, Ontario, Canada). Insulin, glycerol, ethylenediaminetetra-acetic acid (EDTA), dithiothreitol (DTT), bromophenol blue, Na-deoxycholate, Nonidet NP-40, protease inhibitors (EDTA-free), Tween 20, ammonium persulfate (APS), N,N, $\mathrm{N}^{\prime} \mathrm{N}^{\prime}$-tetramethylethylene-diamine (TEMED) were obtained from Sigma-Aldrich (St. Louis, MO, USA). Tris base, $\mathrm{NaCl}$, sodium dodecyl sulfate (SDS), bovine serum albumin (BSA) were purchased from Bioshop Canada Inc. (Burlington, ON, Canada). Methanol and $\mathrm{HCl}$ were obtained from Avantor Performance Materials (Gliwice, Poland). WesternBright ${ }^{\mathrm{TM}}$ Sirius Western blotting detection kit was obtained from Advansta (Menlo Park, CA, USA). A Bradford protein assay kit was obtained from Bio-Rad Laboratories (Hercules, CA, USA). Polyvinylidene difluoride (PVDF) membrane was purchased from Merck Millipore (Darmstadt, Germany). Marker of electrophoresis Thermo Scientific Page Ruler Prestained Protein Ladder was purchased from Thermo Fisher Scientific (Waltham, MA, USA). Antibodies were listed in Table 1.

\section{Cell culture}

BeWo (cat. CCL-98, ATCC, Manassas, VA, USA) passages 18-22, JEG-3 (cat. HTB-36, ATTC) passages 1-6, and H295R (cat. 300483, CLS, Eppelheim,
Germany) passages 17-21 were used in the experiments. All cell lines were cultured in DMEM/F12 without phenol red, supplemented for BeWo cells with: $0.01 \mathrm{mg} / \mathrm{ml}$ insulin and $10 \%$ heat-inactivated FBS, with $10 \%$ heat-inactivated FBS for JEG-3 cells and with $2.5 \%$ NuSerum and $1 \%$ ITS + Premix for H295R cells. BeWo cells have been cultured as nondifferentiated (BeWo) and differentiated (sync. BeWo) cells. Differentiation was achieved using $50 \mu \mathrm{M}$ forskolin. Dose of forskolin was selected based on preliminary experiment with concentrations of forskolin from 10 to $100 \mu \mathrm{M}$ and data published by Zachariades et al. (2011). To select appropriate dose of forskolin effect on proliferation/cytotoxicity and hormone secretion was examined using Alamar blue assay and ELISA (data not shown). Firstly BeWo were cultured in flasks treated with forskolin for $72 \mathrm{~h}$ and then seeded on a plate like syncBeWo. Differentiation markers like syncytin-1 mRNA expression and hCG secretion were chosen based on data previously published by Huang et al. (2009) and analyzed before and after trypsinization. Moreover, viability was measured using Alamar Blue test.

\section{Experimental procedure}

H295R, BeWo, syncBeWo or JEG-3 $\left(12.5 \times 10^{4}\right.$ cells $/ 200 \mu \mathrm{l} /$ well in 96 -well) were cultured alone for 24, 48, and $72 \mathrm{~h}$. At the end of culture, media were frozen at $-20{ }^{\circ} \mathrm{C}$ for further hormone levels analysis while cells were lysed with lysis buffer, sonicated and centrifuged at $15,000 \times g$ for $15 \mathrm{~min}$ at $4{ }^{\circ} \mathrm{C}$ and stored at $-20{ }^{\circ} \mathrm{C}$ for Western blot analysis.

In co-culture BeWo, syncBeWo or JEG-3 cells $\left(1.25 \times 10^{4}\right.$ cells $/ 200 \mu \mathrm{l} /$ well $)$ were cultured on $0.4 \mu \mathrm{m}$ pores/insert with $\mathrm{H} 295 \mathrm{R}$ cells $\left(2.5 \times 10^{4}\right.$ cells/800 $\mu \mathrm{l} /$ well) in 24-well for $72 \mathrm{~h} .24 \mathrm{~h}$ after seeding inserts with BeWo or JEG-3 cells were placed into the wells with H295R cells and media were replaced with fresh H295R cell medium. Hormone levels in medium were evaluated after 24,48 , and $72 \mathrm{~h}$. For co-culture, medium was pooled from inserts and wells. At the end of the incubation period, the cells from inserts (BeWo or JEG-3) were washed with icecold PBS, lysed with lysis buffer, sonicated and centrifuged at $15,000 \times g$ for $15 \mathrm{~min}$ at $4{ }^{\circ} \mathrm{C}$ and stored at $-20{ }^{\circ} \mathrm{C}$ for Western blot analysis. Protein expression was determined in placenta cells (from wells for monoculture or inserts for co-culture) (Fig. 1) 
Table 1 Primary and secondary antibodies

\begin{tabular}{|c|c|c|c|}
\hline Name & Cat no. & Dillution & Company \\
\hline \multicolumn{4}{|l|}{ Primary antibodies } \\
\hline $3 \beta$ HSD goat polyclonal & \#30820 & $1: 200$ & Santa Cruz Biotechnology (Danvers, MA, USA) \\
\hline CYP19 goat polyclonal & \#14244 & & \\
\hline CYP1A1 goat polyclonal & \#9828 & & \\
\hline COMT rabbit polyclonal & \#25844 & & \\
\hline AhR goat polyclonal & \#8088 & & \\
\hline $\mathrm{ER} \alpha$ rabbit polyclonal & \#544 & & \\
\hline $\mathrm{ER} \beta$ rabbit polyclonal & \#8974 & & \\
\hline PR A/B rabbit polyclonal & \#3176S & & \\
\hline$\beta$-actin rabbit monoclonal & \#A5316 & $1: 2000$ & Sigma Aldrich (St. Louis, MO, USA) \\
\hline \multicolumn{4}{|l|}{ Secondary antibodies } \\
\hline Anti-goat horseradish peroxidase-conjugated & \#sc2020 & $1: 1000$ & Santa Cruz Biotechnology (Danvers, MA, USA) \\
\hline Anti-rabbit horseradish peroxidase-conjugated & $\# 7074$ & $1: 1000$ & $\begin{array}{l}\text { Cell Signaling Technologies (Leiden, } \\
\text { Netherlands) }\end{array}$ \\
\hline
\end{tabular}

Cell density for both types of culture was determined experimentally in preliminary studies with $3-15 \times 10^{4}$ for placental cells and $20-100 \times 10^{4}$ for adrenal cells considering size of the plates, time of culture, cell proliferation and hormone secretion.

\section{Real time PCR}

After 24 and $72 \mathrm{~h}$ of incubation with PAHs, the cells were washed with ice-cold PBS, and the plates were frozen at $-20{ }^{\circ} \mathrm{C}$ until needed for the performance of PCR analysis. Total RNA isolation and cDNA synthesis was performed using the TaqMan Gene Expression Cell-to-CT Kit (Applied Biosystems, Carlsbad, CA, USA) following the manufacturer's protocol. The purity and quantity of the RNA and cDNA were determined using spectrophotometry at two optical densities, 260 and $280 \mathrm{~nm}$ (DeNovix DS11 Spectrophotometer, Wilmington, DE, USA). Amplifications were performed using the StepOnePlus system (Applied Biosystems, Carlsbad, CA, USA) and the TaqMan syncytin-1 in combination with the TaqMan Gene Expression Master Mix (Applied Biosystems), following the manufacturer's instructions. PCR was performed using a final volume of $20 \mu \mathrm{l}$, including $50 \mathrm{ng} /$ reaction cDNA. The PCR conditions were performed as follows: pre-incubation for $2 \mathrm{~min}$ at $50{ }^{\circ} \mathrm{C}$ and $10 \mathrm{~min}$ at $95{ }^{\circ} \mathrm{C}$, amplification for 40 cycles $\left(15 \mathrm{~s}\right.$ at $95^{\circ} \mathrm{C}$ and $1 \mathrm{~min}$ at $\left.60^{\circ} \mathrm{C}\right)$. The relative expression of genes was normalized against the endogenous reference gene GAPDH (Human GAPD Endogenous Control, Number 4333764F); $\Delta \mathrm{C}_{\mathrm{q}}$ ) and converted to relative expression (RQ) using the $2^{-\Delta \Delta \mathrm{Cq}}$ method. The results are expressed in the figures as relative values (RQ).

Alamar blue assay

The alamarBlue assay (Invitrogen) is designed to measure the proliferation of different cell types and is based on quantitation of the cell's metabolic activity. Cellular metabolism induces a chemical reduction of the alamarBlue medium, i.e., this assay is based on the quantitative metabolic conversion of the blue, nonfluorescent resazurin to pink, fluorescent resorufin by living cells. AlamarBlue has minimal cell toxicity and is therefore appropriate for continuous monitoring of proliferation in the same cell culture at various time points. AlamarBlue stock solution was aseptically added to the wells in amounts equal to $10 \%$ of the incubation volume. The resazurin reduction in the cultures after 24 and $72 \mathrm{~h}$ of treatment with PAHs was determined after $3 \mathrm{~h}$ incubation with alamarBlue by measuring the absorbance at $570 \mathrm{~nm}$ and $600 \mathrm{~nm}$ wavelengths using a FLUORO microplate reader (BioTek Instruments, Winooski, VT, USA). The results are expressed in the figures as relative fluorescence units (RFU). 


\section{TIME OF CULTURE}

$\underbrace{0-24 \mathrm{~h}}_{\begin{array}{c}\text { CELL PLATING AND } \\ \text { CELL ATTACHMENT }\end{array}} \underbrace{78 \mathrm{~h}}_{\text {CELL CULTURE AND BIOCHEMICAL ASSAYS }} \underbrace{72 \mathrm{~h}}$

Each cells were seeded in seperate plate in their regular medium.

After $24 \mathrm{~h}$ the regular medium was replaced with fresh regular medium for monoculture or co-culture medium for co-cultures.
After each time point medium was removed from wells for monoculture or pooled from insert and well for coculture prior to analysis hormone secretion. Placenta cells were removed from inserts or wells and each cell type were analyzed seperatly by Western blott.

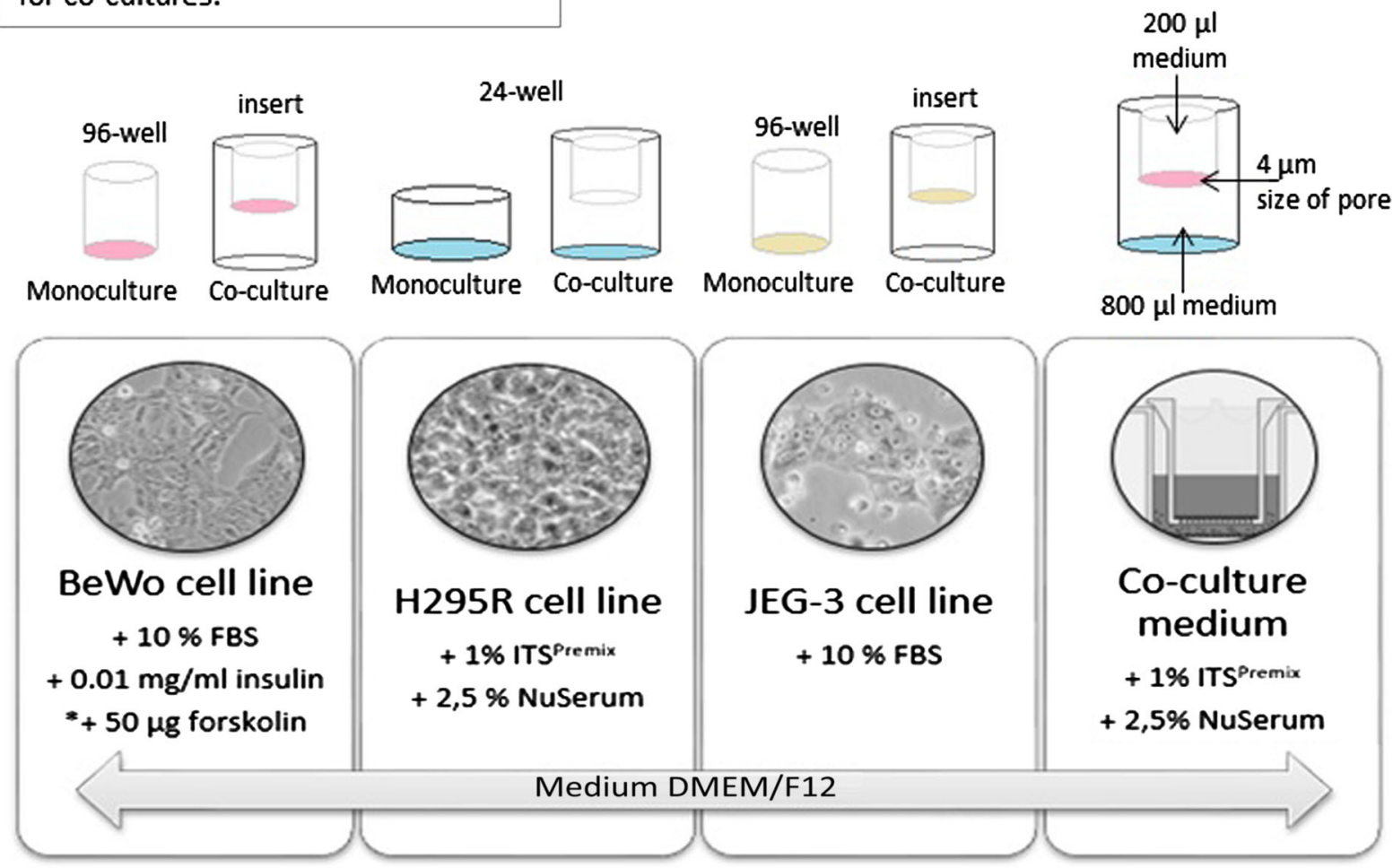

Fig. 1 Experimental procedure

ELISA

P4, E2 and hCG levels were determined using commercially available ELISA kits (cat. EIA-1561, EIA-2693 and EIA-1911, respectively, DRG Instruments GmbH, Marburg, Germany). For P4 the sensitivity of assay was $0.045 \mathrm{ng} / \mathrm{ml}$. The inter- and intraexperiment coefficients of variation were 4.34 and $6.99 \%$, respectively. Cross reactivity with $\mathrm{P} 4: 100 \%$, $17 \alpha \mathrm{OH}$ progesterone: $0.30 \%$, pregnenolone: $0.35 \%$, estriol: $<0.10 \%$ and $17 \alpha$ estradiol: $<0.10 \%$. For E2 the sensitivity of assay was $9.714 \mathrm{pg} / \mathrm{ml}$. The inter- and intra-experiment coefficients of variation were 6.72 and $2.71 \%$, respectively. Cross reactivity of the E2 assay were tested for E2: $100 \%$, estriol: $0.05 \%$, estrone: $0.2 \%, \mathrm{P} 4: 0 \%$ and pregnenolone $0 \%$. For hCG the sensitivity of assay was $1 \mathrm{mlU} /$ with ranges of 0-200 $\mathrm{mlU} / \mathrm{ml}$. The inter- and intra-experiment coefficients of variation were 7.3 and $4 \%$, respectively. 
Western blot

$80 \mu \mathrm{g}$ of protein (determined by the Bradford method) from each treatment group were separated by $10 \%$ SDS-PAGE and transferred to polyvinylidene difluoride membranes. The blots were blocked for $1 \mathrm{~h}$ in $5 \%$ w/v BSA and $0.1 \% \mathrm{v} / \mathrm{v}$ Tween 20 in $0.02 \mathrm{M}$ TBS buffer and incubated with primary. The blots were then incubated with primary antibodies listed in Table 1 diluted 1:200 at $4{ }^{\circ} \mathrm{C}$ overnight and then with a horseradish peroxidase-conjugated secondary antibody diluted 1:1000. After visualization, blots were stripped for $30 \mathrm{~min}$ using stripping buffer (glycine, $\mathrm{HCl} 1 \%$ SDS), next blocked $1 \mathrm{~h}$ in 5\% w/v BSA and $0.1 \% \mathrm{v} / \mathrm{v}$ Tween 20 in $0.02 \mathrm{M}$ TBS buffer and incubated with primary antibodies for anti- $\beta$-actin diluted 1:2000 at $4{ }^{\circ} \mathrm{C}$ overnight and then with a horseradish peroxidase-conjugated secondary antibody diluted 1:1000. Signals were detected by chemiluminescence using a WesternBright ${ }^{\mathrm{TM}}$ Sirius, and visualized using a ChemiDoc-It Imaging System.

Statistical analysis

All samples were analyzed in triplicate in the same assay and all assays were repeated three times $(\mathrm{n}=3)$. Experimental results are presented as mean \pm S.D. All statistical analyses were performed using Graph Pad Prism 5. The data were analyzed by one- or twoway ANOVA followed by Tukey's honest significant differences multiple comparison test. Statistical significant differences between time of culture and mono versus coculture are indicated with different letters a;b;c;d;e $(P<0.05)$, the same letters indicating no significant differences.

\section{Results}

Syncytin-1 gene expression in BeWo and syncBeWo before and after trypsynization

Before trypsynization, forskolin increased 23 fold syncytin-1 gene expression $(P<0.001)$ (Table 1 , Suppl). After trypsynization syncytin-1 gene expression was 37 fold higher compared to BeWo not treated with forskolin $(P<0.001)$ (Table 1 in Supplementary).
Effect of trypsynization on syncBeWo cell's viability

24 and $72 \mathrm{~h}$ after trypsynization, in both, monoculture of syncBeWo and co-culture of syncBeWo with H295R cells no effect on cell proliferation was observed (Fig. 1 Suppl).

Hormone secretion and steroidogenic enzymes expression

H295R cells secreted low amount of P4 (Fig. 2A, C, E) and E2 (Fig. 3A, C, E). In co-cultures of JEG-3 and BeWo with H295R cells' P4 secretion was higher than in the monoculture while in syncBeWo lower versus monoculture $(P<0.05)$ (Fig. $2 \mathrm{~A}, \mathrm{C}, \mathrm{E})$. A similar pattern was observed for $3 \beta \mathrm{HSD}$ protein expression in mono- but not in co-culture with JEG-3 cells $(P<0.05)$ (Fig. 2B) and in both mono and co-culture with BeWo $(P<0.05)$ (Fig. 2D). In syncBeWo an increase in $3 \beta \mathrm{HSD}$ protein expression in monoculture $(P<0.05)$ and no difference in co-culture was noted (Fig. 2F).

E2 secretion in monoculture of all JEG-3, BeWo and sync BeWo increased with time of culture. In JEG-3 with H295R cells co-culture 17, 27 and 21-fold higher levels of E2 were observed after 24, 48 and $72 \mathrm{~h}$, respectively, and was paralleled with increased CYP19 protein expression $(P<0.05)$ (Fig. 3B). In the BeWo co-culture model a time dependent effect was noted compared to monoculture: both E2 secretion and CYP19 expression decreased after $24 \mathrm{~h}$ of culture, increased E2 secretion with decreased CYP19 expression after $48 \mathrm{~h}$ of culture and 23-fold higher E2 secretion corresponding with increased CYP19 expression after $72 \mathrm{~h}$ of culture was noted (Fig. 3C, D). In syncBeWo cells co-culture, E2 levels significantly increased and was 3.2, 10, and 25-fold higher after 24,48 and $72 \mathrm{~h}$, respectively, versus monoculture $(P<0.05) \quad$ (Fig. 3E). Significantly increased CYP19 protein expression in both mono- (after $48 \mathrm{~h}$ ) and co-culture (after $72 \mathrm{~h})$ was observed $(P<0.05)$ (Fig. 3F).

Levels of hCG significantly increased with time of culture in monoculture of all investigated cell lines (Fig. 4). There was no change in levels with time in co-culture of JEG-3 with H295R, 3.0, 1.4 and 2.5-fold higher after 24,48 and $72 \mathrm{~h}$, respectively, of coculture of BeWo with H295R $(P<0.05)$ (Fig. 4A, B), 
Fig. 2 P4 secretion and $3 \beta$ HSD protein expression in JEG-3 (A, B), BeWo (C, D) and syncBeWo $(\mathbf{E}$, F) mono- and co-cultures with H295R. The protein levels of $3 \beta \mathrm{HSD}(42 \mathrm{kDa})$ were densitometrically scanned and normalised against the $\beta$-actin $(42 \mathrm{kDa})$ signal. ELISA and Western blotting experiments were independently performed and repeated three times $(\mathrm{n}=3)$. The data are plotted as the mean \pm S.D.

Statistical significant differences between time of culture and mono- versus co-culture are indicated with different letters $(P<0.05)$, the same letters indicating no significant differences
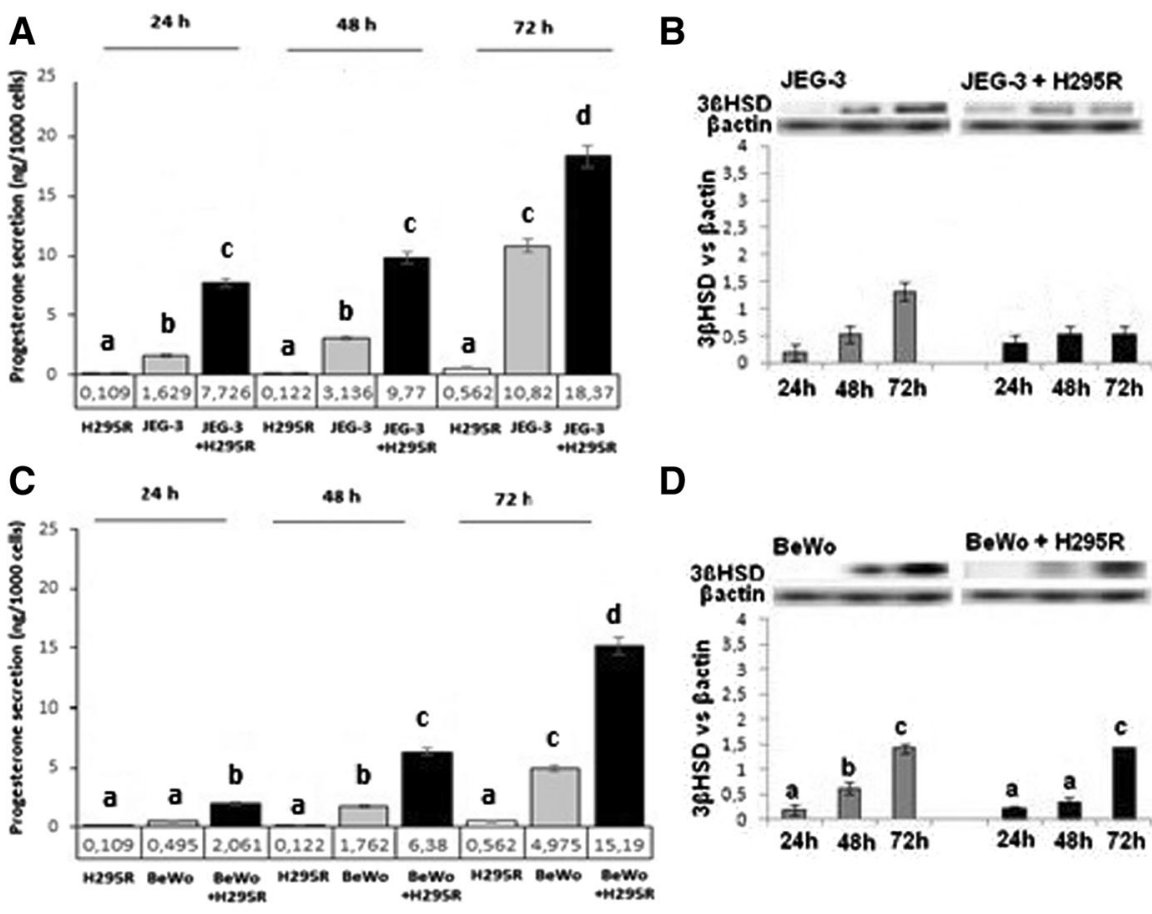

E

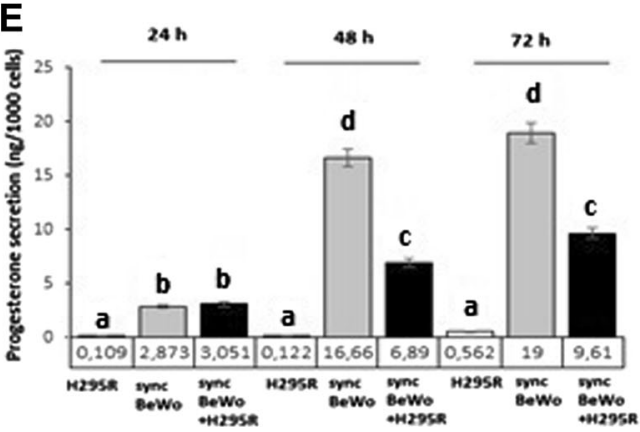

$\mathbf{F}$

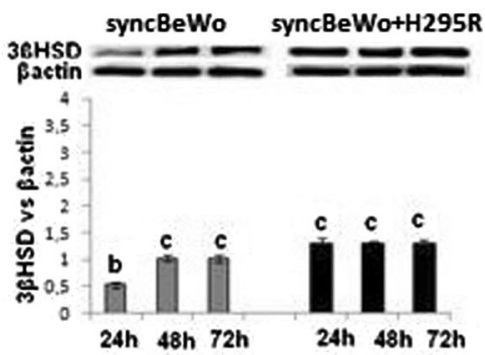

while in co-culture of sync BeWo with H295R 5.9, 7.1 and 1.2-fold lower levels were observed at 24, 48 and $72 \mathrm{~h}$, respectively $(P<0.05)$ (Fig. 4C).

$\mathrm{PR}$ protein expression in monoculture was higher in BeWo than in JEG-3 cells and higher in BeWo than in syncBeWo cells. The profile of $\mathrm{PR}$ protein expression, in both mono and co-culture of JEG-3 and BeWo cells, increased during time of cell culture, while no changes were observed in syncBeWo cells $(P<0.05)$ (Fig. 5A-C). Interestingly, although antibodies against total PR were used, in fully syncytialised BeWo cells (treated with $50 \mu \mathrm{M}$ forskolin for $72 \mathrm{~h}$ ) two forms of PR $(A+B)$ were observed.

The profile of $\mathrm{ER} \alpha$ protein expression increased with time of culture $(P<0.05)$ (Fig. 5D-F) in all tested models; however, expression was significantly higher in syncBeWo cells in mono- and co-culture with H295R cells $(P<0.05)$ (Fig. 4F). The profile of ER $\beta$ in mono- and co-culture of JEG-3 and BeWo cells was comparable and increased with time of culture, while in syncBeWo cells there was no change in levels during all times of culture. $(P<0.05)$ (Fig. 5G-I).

The expression of AhR in JEG-3 cells increased with time of culture both in mono- and co-culture with $\mathrm{H} 295 \mathrm{R}$ cells and reached the highest level after $72 \mathrm{~h}$ of culture (Fig. 6A). In monoculture of BeWo cells, AhR protein expression decreased after $72 \mathrm{~h}$ of culture while in co-culture expression was lower at $24 \mathrm{~h}$ in comparison with monoculture, and at the same levels at 48 and $72 \mathrm{~h}$, as in monoculture $(P<0.05)$ (Fig. 6B). In monoculture of syncBeWo cells, AhR protein expression increased with time of culture and 
Fig. 3 E2 secretion and CYP19 protein expression in JEG-3 (A, B), BeWo $(\mathbf{C}$, D) and syncBeWo $(\mathbf{E}, \mathbf{F})$ mono- and co-cultures with H295R. The protein levels of CYP19 (50 kDa), were densitometrically scanned and normalised against the $\beta$-actin (42 kDa) signal. ELISA and Western blotting experiments were independently performed and repeated three times $(\mathrm{n}=3)$. The data are plotted as the mean \pm S.D.

Statistical significant differences between time of culture and mono versus coculture are indicated with different letters $(P<0.05)$, the same letters indicating no significant differences
A

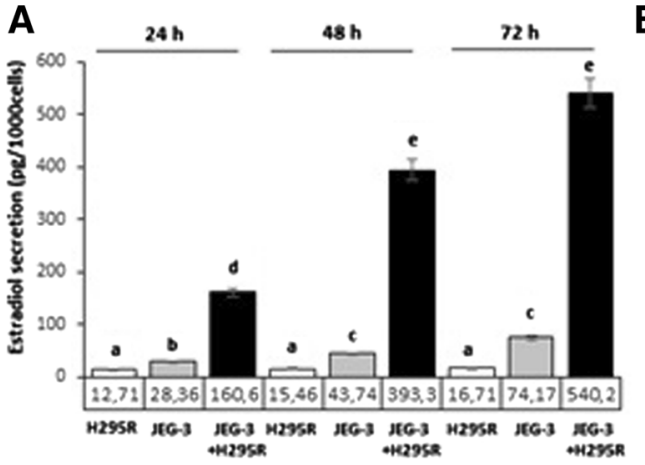

C

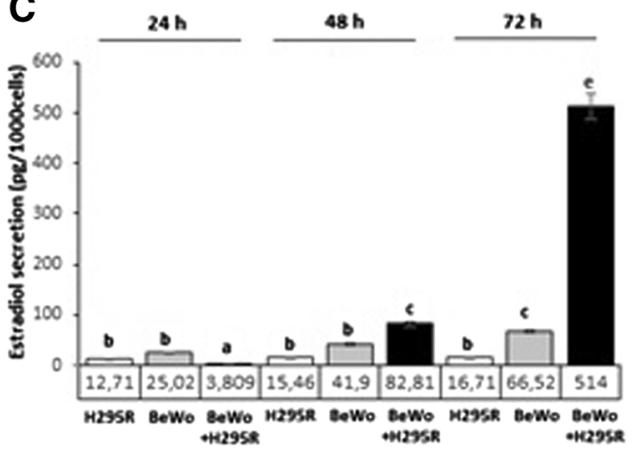

E

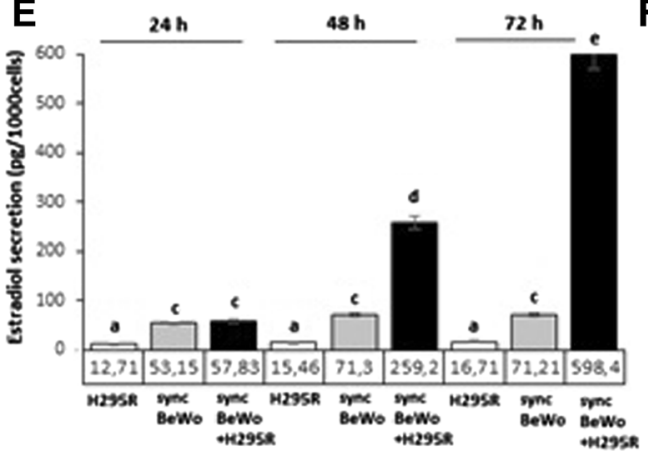

B

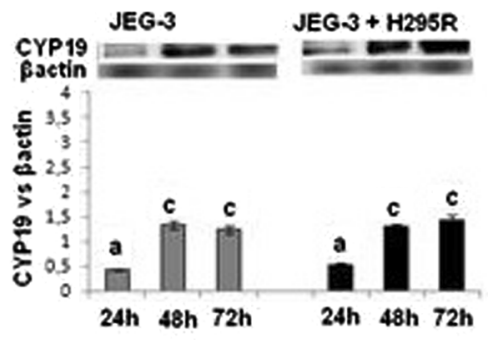

D

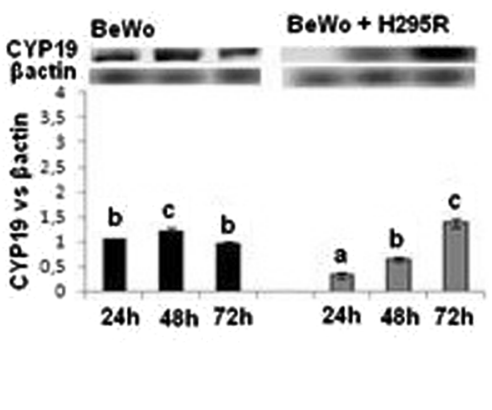

$\mathbf{F}$

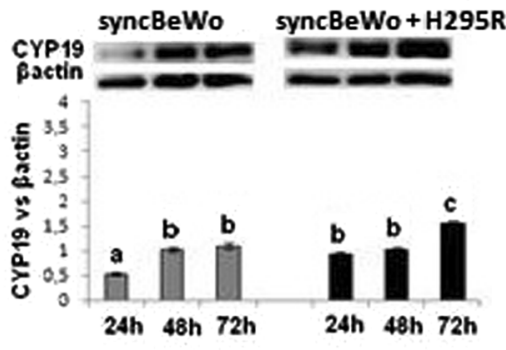

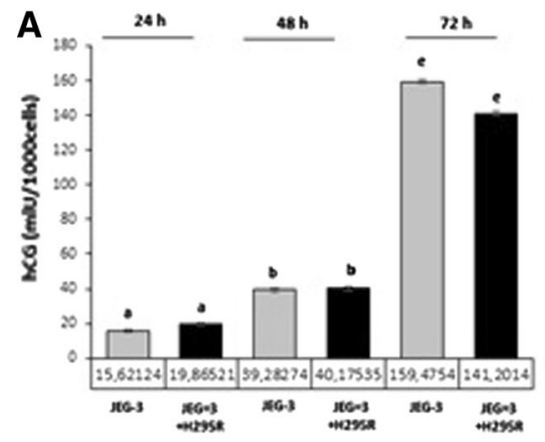

B

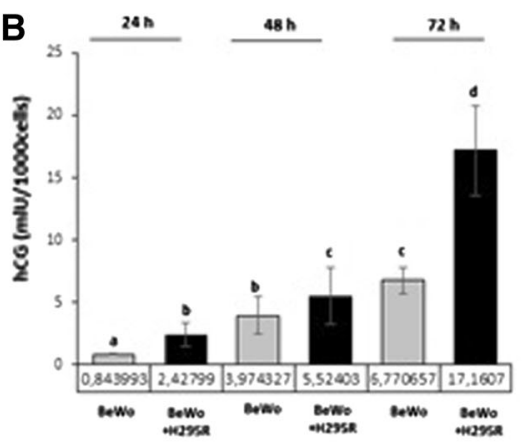

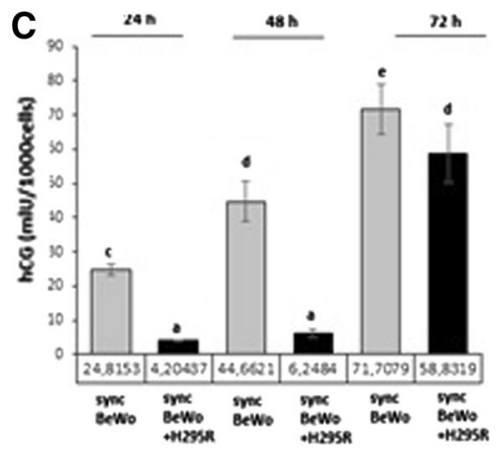

Fig. 4 hCG secretion in JEG-3 (A), BeWo (B) and syncBeWo (C) in mono- and co-cultures with H295R. The data are plotted as the mean \pm SD. Statistical significant differences between time of culture and mono versus coculture are indicated with different letters $(P<0.05)$, the same letters indicating no significant differences 

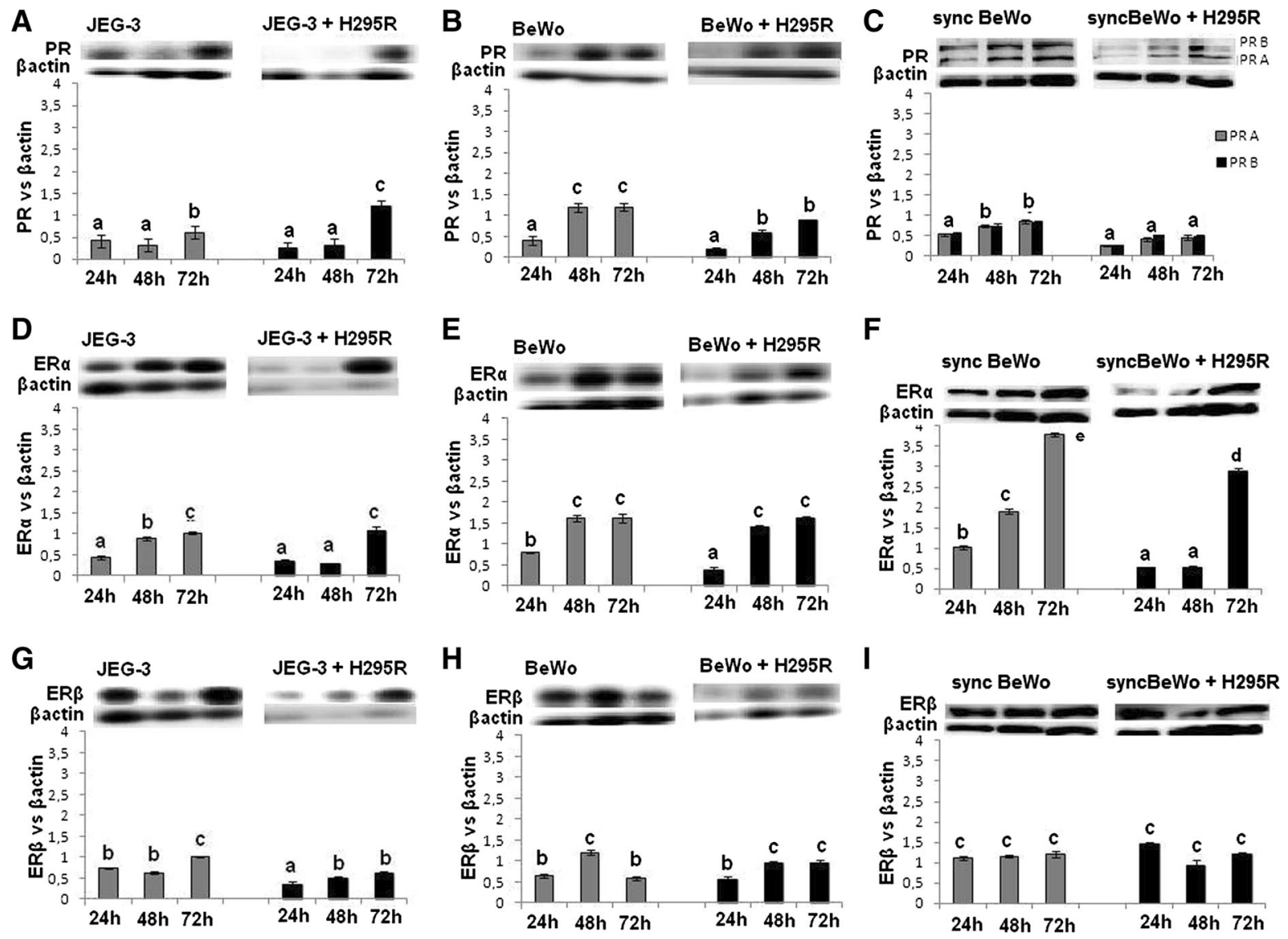

Fig. 5 Expression of PR A/B and ER $\alpha / \beta$ in JEG-3 (A, D, G), BeWo $(\mathbf{B}, \mathbf{E}, \mathbf{H})$ and syncBeWo $(\mathbf{C}, \mathbf{F}, \mathbf{I})$ in mono- and cocultures with H295R. The protein levels of PR (PR A $118 \mathrm{kDa}$; PR B $90 \mathrm{kDa}), \mathrm{ER} \alpha(65 \mathrm{kDa})$, and $\mathrm{ER} \beta(55 \mathrm{kDa})$ were densitometrically scanned and normalised against the $\beta$-actin $(42 \mathrm{kDa})$ signal. Western blotting experiments were

was significantly higher than in monoculture of BeWo cells. In co-culture of syncBeWo cells with H295R cells, high expression was noted during all times of culture and this was twofold higher than in co-culture of BeWo cells with H295R $(P<0.05)$ (Fig. 6C).

The profile of CYP1A1 protein expression in monoculture and co-culture with H295R cells in JEG-3 cells and BeWo cells was comparable, 2.5 to threefold higher expression was noted in syncBeWo cells in mono and co-culture $(P<0.05)$ (Fig. 6D-F). In monoculture of all cell types we noted low protein expression of COMT. Expression of COMT was slightly increased at $72 \mathrm{~h}$ of co-culture in BeWo cells with $\mathrm{H} 295 \mathrm{R}$ and was the highest in co-culture of syncBeWo cells with H295R (Fig. 6G-I). independently performed and repeated three times $(n=3)$. The data are plotted as the mean \pm S.D. Statistical significant differences between time of culture and mono- versus coculture are indicated with different letters $(P<0.05)$, the same letters indicating no significant differences

\section{Discussion}

In the presented data we compare different steroidogenic and metabolic parameters in co-cultures of JEG3, BeWo and syncBeWo placental cell lines with an adrenal cell line (H295R).

By comparing co-cultures of placental cells we showed that both $\mathrm{P} 4$ and E2 secretion increased in JEG-3 and BeWo while in syncBeWo lower P4 secretion was noted. Lower levels of $\mathrm{P} 4$ secretion noted in co-culture of syncBeWo cells could be explained by the high levels of E2 secreted by the syncBeWo cells. It is well known that P4 levels during placentation decrease in favor of increased E2 secretion (Hill 2014) and diminished hCG production (Aspillaga et al. 1983), which we also observed in our 

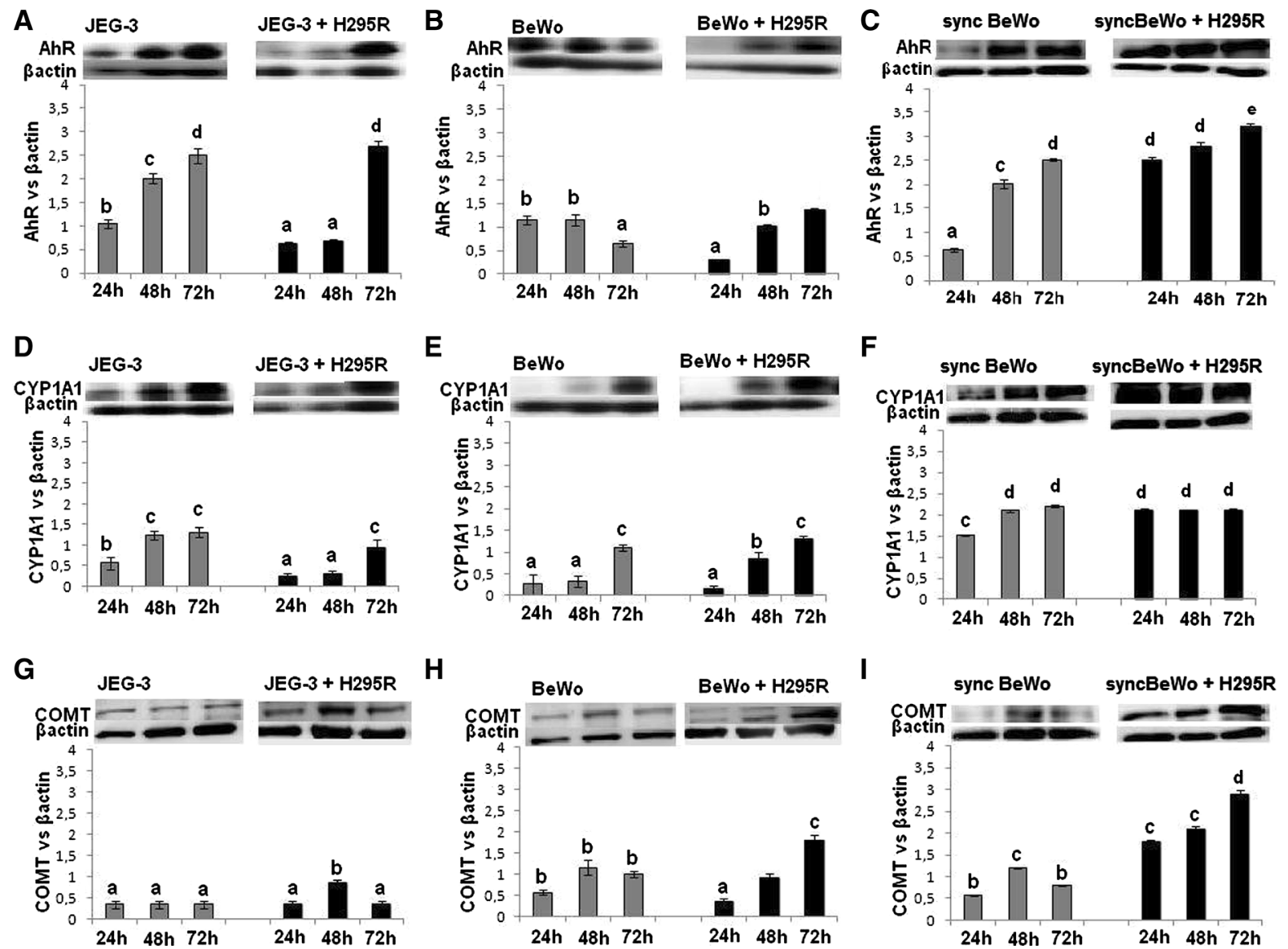

Fig. 6 Protein expression of AhR, CYP1A1 and COMT in JEG-3 (A, D, G), BeWo $(\mathbf{B}, \mathbf{E}, \mathbf{H})$ and syncBeWo $(\mathbf{C}, \mathbf{F}, \mathbf{I})$ in mono- and co-culture with H295R. The data are plotted as the mean \pm SD. The protein levels of AhR $(90 \mathrm{kDa})$, CYP1A1 (56 kDa), COMT (25 kDa) were densitometrically scanned and normalised against the $\beta$-actin (42 kDa) signal. Western blotting

study. Although the protein expression profile was similar in all tested models, the highest expression of $3 \beta \mathrm{HSD}$ was observed in co-culture of syncBeWo cells. It is well known that $3 \beta \mathrm{HSD}$ enzymes operate during placental cell differentiation (Meeker et al. 1971).

E2 secretion was significantly higher in all placental co-culture models compare to monocultures. The higher levels of E2 secretion can be explained with increased CYP19 protein expression in all co-culture models at all time points. Observed by us increased synergistically E2 secretion in co-culture of H295R and BeWo after $72 \mathrm{~h}$ of culture cells is in accordance with previously published data (Thibeault et al. 2014). It is also in agreement with data by Hill (2014), experiments were independently performed and repeated three times $(n=3)$. Statistical significant differences between time of culture and mono- versus coculture are indicated with different letters $(P<0.05)$, the same letters indicating no significant differences

who showed that progression of development and differentiation of the placenta is connected with an increase of placental capacity for the aromatization of androgens, and therefore with an increase of CYP19 expression or aromatase activity.

Differences in hormone secretion observed in coculture models were results from the interaction between placental and adrenal. For estrogen synthesis, in contrast to other steroidogenic organ, the placenta does not express the cytochrome P450 17 $\beta$-hydroxylase-17:20 lyase, and therefore cannot convert pregnenolone and progesterone into androgens. Thus the production of placental estrogens is tributary of a precursor androgen, the sulphate of 
dehydroepiandrosterone (S-DHA) produced by the maternal and adrenal gland (Thibeault et al. 2014).

We have demonstrated the cell-specific secretion of hCG in both mono- and co-cultures. The highest level of secretion of this hormone was noted in the JEG-3 cell; however, there was no difference between secretion by mono- and co-culture. It has previously been shown that a large amount of hCG, directly involved in the quality of placentation, is secreted by extravillous trophoblasts during the first trimester of pregnancy and likely hyperglycosylated hCG secretion promotes trophoblast invasion (Handschuh et al. 2007), thus confirming our results. Additionally, we showed that syncBeWo cells secreted four times more hCG than BeWo cells which corresponded with lower $\mathrm{P} 4$ levels. As we discussed above, during placentation P4 levels decrease in favor of an increase in E2 secretion (Hill 2014) and diminished hCG production (Aspillaga et al. 1983).

No differences between PR expression in JEG-3 and BeWo co-culture, while statistically significant lower PR expression in co-cultures of syncBeWo cells were observed. The profile of PR protein expression, in co-culture of JEG-3 and BeWo, increased over time of cell culture, while there was no change in syncBeWo cells. Lower PR expression noted in coculture of syncBeWo cell could be explained by lower P4 secretion corresponding with high levels of E2 secretion and ER expression. Zachariades et al. (2011) suggested that PR is implicated in the developmental events leading to placental syncytialisation. Our observation of the downregulation of PR expression in syncBeWo cell versus BeWo cells could be justified by the fact that these cells are fully syncytialised, thus resembling a third trimester placental model (Zachariades et al. 2011).

From our observation of ER, all used placental cell lines had higher expression of ER $\alpha$ than ER $\beta$. We noted no significant difference between monoculture and co-culture of JEG-3 and BeWo cell models although there was higher ER $\alpha$ expression in syncBeWo. This finding is in agreement with the data of Bukovsky et al. (2003) who showed similar relationships in placental explants. Bukovsky et al. (2003) documented that E2 plays a role in the stimulation of terminal differentiation of mononucleated cytotrophoblast cells and promotes placental function, via the $E R \alpha$. An increase of ER $\alpha / \beta \gamma$ mRNA in the placenta throughout gestation was reported by Fujimoto et al. (2005).

The next important problem discussed in this study relates to receptor and enzymes involved in the metabolism of different exogenous and endogenous compounds. The AhR acts as a xenobiotic receptor for a number of different xenobiotics, thus playing a critical role in human placenta (Stejskalova and Pavek 2011). We observed statistically significant differences in AhR expression between the used models with the highest expression in co-culture of syncBeWo. Taking into account that there are studies which show multiple mechanisms of AhR-ER (Helle et al. 2016; Barć and Gregoraszczuk 2016) we suggest that this difference between the types of model is attributed to higher ER expression in syncBeWo. The consequence of AhR receptor stimulation is the activation of detoxifying enzymes of phase I (CYP1A1) and phase II (i.e. COMT) of metabolism. In the present study, we showed comparable CYP1A1 and COMT expression in JEG-3 and BeWo cells, while higher expression was observed in syncBeWo cells, both in mono- and coculture. CYP1A1 expression and activity have been detected in the first and third trimesters at both mRNA and protein levels (Hakkola et al. 1996a, b; Stejskalova and Pavek 2011; Czekaj et al. 2005). Palmer et al. (2011) analyzed COMT expression showed that it was mainly expressed in the syncytiotrophoblast, which confirms our result of a higher expression of COMT in syncBeWo. Higher expression of both CYP1A1 and COMT in syncBeWo cells could be additionally explained by the fact that both enzymes are important for the metabolism of estrogens (Parl et al. 2009; Mannisto and Kaakkola 1999), which were in our coculture model produced at the highest amount in syncBeWo cells compared with JEG-3 and BeWo cells. The exact molecular mechanism underlying the difference between unsyncytialised and syncytialised cells needs further study however it was not a scope of our study.

\section{Conclusion}

Based on our results we conclude that, the difference in hCG secretion between JEG-3 and BeWo representing villous and extravillous phenotype could be used to study fetoplacental steroidogenesis in 1st and 3rd trimester, respectively. In turn syncBeWo model 
exhibiting the highest expression of AhR, CYP1A1 and COMT could be suitable to study the metabolism. However, taking into consideration that in the development and function of the human placenta participate numerous factors we must keep in mind the limitation of each experimental model.

Acknowledgements This work was supported partly by the Jagiellonian University in Krakow K/ZDS/005404 and K/DSC/ 003952 and partly by National Sciences Centre, Poland 2015/19/N/NZ7/01093. The present publication contains part of Eliza Drwal Ph.D. dissertation.

\section{Compliance with ethical standards}

Conflict of interest The authors declare no conflict of interest.

Open Access This article is distributed under the terms of the Creative Commons Attribution 4.0 International License (http:// creativecommons.org/licenses/by/4.0/), which permits unrestricted use, distribution, and reproduction in any medium, provided you give appropriate credit to the original author(s) and the source, provide a link to the Creative Commons license, and indicate if changes were made.

\section{References}

Aspillaga MO, Whittaker PG, Grey CE, Lind T (1983) Endocrinologist events in early pregnancy failure. Am J Obstet Gynecol 15:903-908

Barć J, Gregoraszczuk EL (2016) Halowax 1051 affects steroidogenesis by down-regulation of aryl hydrocarbon and estrogen receptors and up-regulation of androgen receptor in porcine ovarian follicles. Chemosphere 144:467-474

Barnea ER, Maclusky NJ, Decherney Naftolin F (1988) Catechol-o-methyl transferase activity in the human term placenta. Am J Perinato 5:121-127

Bláha L, Hilscherová K, Mazurová E, Hecker M, Jones PD, Newsted JL, Bradley PW, Gracia T, Duriš Z, Horká I, Holoubek I, Giesy JP (2006) Alteration of steroidogenesis in H295R cells by organic sediment contaminants and relationships to other endocrine disrupting effects. Environ Int 32:749-757

Bukovsky A, Cekanova M, Caudle MR, Wimalasena J, Foster JS, Henley DC, Elder RF (2003) Expression and localization of estrogen receptor-alpha protein in normal and abnormal term placentae and stimulation of trophoblast differentiation by estradiol. Reprod Biol Endocrinol 6:1-13

Burleigh DW, Kendziorski CM, Choi YJ, Grindle KM, Grendell RL, Magness RR, Golos TG (2007) Microarray analysis of BeWo and JEG3 trophoblast cell lines: identification of differentially expressed transcripts. Placenta 28:383-389

Czekaj P, Wiaderkiewicz A, Florek E, Wiaderkiewicz R (2005) Tobacco smoke-dependent changes in cytochrome $\mathrm{P} 450$
1A1, 1A2, and 2E1 protein expressions in fetuses, newborns, pregnant rats, and human placenta. Arch Toxicol 79:13-24

Dunk C, Petkovic L, Baczyk D, Rossant J, Winterhager E, Lye S (2003) A novel in vitro model of trophoblast-mediated decidual blood vessel remodeling. Lab Invest 83: $1821-1828$

Escobar JC, Patel SS, Beshay VE, Suzuki T, Carr BR (2011) The human placenta expresses CYP17 and generates androgens de novo. J Clin Endocrinol Metab 96:1385-1392

Frendo JL, Olivier D, Cheynet V, Blond JL, Bouton O, Vidaud M, Rabreau M, Evain-Brion D, Mallet F (2003) Direct involvement of HERV-W Env glycoprotein in human trophoblast cell fusion and differentiation. Mol Cell Biol 23:3566-3574

Fujimoto J, Nakagawa Y, Toyoki H, Sakaguchi H, Sato E, Tamaya T (2005) Estrogen-related receptor expression in placenta throughout gestation. J Steroid Biochem Mol Biol 94:67-69

Gude NM, Roberts CT, Kalionis B, King RG (2004) Growth and function of the normal human placenta. Thromb Res 114:397-407

Hakkola J, Pasanen M, Hukkanen J, Pelkonen O, Mäenpää J, Edwards RJ, Boobis AR, Raunio H (1996a) Expression of xenobiotic-metabolizing cytochrome $\mathrm{P} 450$ forms in human full-term placenta. Biochem Pharmacol 23:403-411

Hakkola J, Raunio H, Purkunen R, Pelkonen O, Saarikoski S, Cresteil T, Pasanen M (1996b) Detection of cytochrome $\mathrm{P} 450$ gene expression in human placenta in first trimester of pregnancy. Biochem Pharmacol 51:379-383

Handschuh K, Guibourdenche J, Tsatsaris V, Guesnon M, Laurendeau I, Evain-Brion D, Fournier T (2007) Human chorionic gonadotropin produced by the invasive trophoblast but not the villous trophoblast promotes cell invasion and is down-regulated by peroxisome proliferator-activated receptor-gamma. Endocrinology 148: 5011-5019

Hannan NJ, Paiva P, Dimitriadis E, Salamonsen LA (2010) Models for study of human embryo implantation: choice of cell lines? Biol Reprod 82:235-245

Helige C, Ahammer H, Hammer A, Huppertz B, Frank HG, Dohr G (2008) Trophoblastic invasion in vitro and in vivo: similarities and differences. Hum Reprod 23:2282-2291

Helle J, Bader M, Keiler AM, Zierau O, Vollmer G, Chittur SV, Tenniswood M, Kretzschmar G (2016) Cross-talk in the female rat mammary gland: influence of aryl hydrocarbon receptor on estrogen receptor signaling. Environ Health Perspect 124:601-610

Hill M (2014) Special issue on pregnancy and steroids: editorial. J Steroid Biochem Mol Biol 139:105-106

Huang FD, Kung FL, Tseng YC, Chen MR, Chan HS, Lin CJ (2009) Regulation of protein expression and function of octn2 in forskolin-induced syncytialization in BeWo cells. Placenta 30:187-194

Isoherranen N, Thummel KE (2013) Drug metabolism and transport during pregnancy: how does drug disposition change during pregnancy and what are the mechanisms that cause such changes? Drug Metab Dispos 41:256-262

Jiang SW, Lloyd RV, Jin L, Eberhardt NL (1997) Estrogen receptor expression and growth-promoting function in human choriocarcinoma cells. DNA Cell Biol 16:969-977 
Lee CQ, Gardner L, Turco M, Zhao N, Murray MJ, Coleman N, Rossant J, Hemberger M, Moffett A (2016) What is trophoblast? A combination of criteria define human firsttrimester trophoblast. Stem Cell Rep 6:257-272

Mannelli C, Ietta F, Avanzati AM, Skarzynski D, Paulesu L (2015) Biological tools to study the effects of environmental contaminants at the feto-maternal interface. Dose Response 13:1559325815611902

Mannisto PT, Kaakkola S (1999) Catechol-O-methyl-transferase (COMT): biochemistry, molecular biology, pharmacology, and clinical efficacy of the new selective COMT inhibitors. Pharm Rev 51:593-628

Meeker C, DeCesaris V, Tulp O (1971) Metabolism of 7-3 $\mathrm{H}$-pregnenolone in normal human placenta maintained in organ culture. Am J Obstet Gynecol 111:840-845

Mehta DV, Kim YS, Dixon D, Jetten AM (2002) Characterization of the expression of the retinoid-related, testis-associated receptor (RTR) in trophoblasts. Placenta 23: 281-287

Moser G, Gauster M, Orendi K, Glasner A, Theuerkauf R, Huppertz B (2010) Endoglandular trophoblast, an alternative route of trophoblast invasion? Analysis with novel confrontation co-culture models. Hum Reprod 25: $1127-1136$

Orendi K, Gauster M, Moser G, Meiri H, Huppertz B (2010) The choriocarcinoma cell line BeWo: syncytial fusion and expression of syncytium-specific proteins. Reproduction 140:759-766

Palmer K, Saglam B, Whitehead C, Stock O, Lappas M, Tong S (2011) Severe early-onset preeclampsia is not associated with a change in placental catechol $O$-methyltransferase (COMT) expression. Am J Pathol 178:2484-2488

Parl FF, Dawling S, Roodi N, Crooke PS (2009) Estrogen metabolism, breast cancer: a risk model. Ann NY Acad Sci 1155:68-75
Stejskalova L, Pavek P (2011) The function of cytochrome P450 1A1 enzyme (CYP1A1) and aryl hydrocarbon receptor $(\mathrm{AhR})$ in the placenta. Curr Pharm Biotechnol 12:715-730

Stejskalova L, Rulcova A, Vrzal R, Dvorak Z, Pavek P (2013) Dexamethasone accelerates degradation of aryl hydrocarbon receptor (AHR) and suppresses CYP1A1 induction in placental JEG-3 cell line. Toxicol Lett 25:183-191

Sullivan MH (2002) Endocrine cell lines from the placenta. Mol Cell Endocrinol 228:103-119

Thibeault AH, Deroy K, Vaillancourt C, Sanderson JT (2014) A unique co-culture model for fundamental and applied studies of human fetoplacental steroidogenesis and interference by environmental chemicals. Environ Health Perspect 122:371-377

Tsampalas M, Gridelet V, Berndt S, Foidart JM, Geenen V, Perrier d'Hauterive S (2010) Human chorionic gonadotropin: a hormone with immunological and angiogenic properties. J Reprod Immunol 85:93-98

Wang H, Pilla F, Anderson S, Martínez-Escribano S, Herrer I, Moreno-Moya JM, Musti S, Bocca S, Oehninger S, Horcajadas JA (2012) A novel model of human implantation: 3D endometrium-like culture system to study attachment of human trophoblast (Jar) cell spheroids. Mol Hum Reprod 18:33-43

Wice B, Menton D, Geuze H, Schwartz AL (1990) Modulators of cyclic AMP metabolism induce syncytiotrophoblast formation in vitro. Exp Cell Res 186:306-316

Zachariades E, Foster H, Goumenou A, Thomas P, RandWeaver M, Karteris E (2011) Expression of membrane and nuclear progesterone receptors in two human placental choriocarcinoma cell lines (JEG-3 and BeWo): effects of syncytialisation. J Mol Med 27:767-774 